\title{
Urethral Duplication with Two Hypospadic Meati-An Unusual Variant
}

\author{
Joseph Rutherford Davidson ${ }^{1} \quad$ Naomi Jane Wright ${ }^{1} \quad$ Massimo Garriboli $^{1}$ \\ 1 Department of Paediatric Urology, Evelina London Children's \\ Hospital, London, United Kingdom \\ Address for correspondence Massimo Garriboli, Department of \\ Paediatric Urology, Evelina London Children's Hospital, London, United \\ Kingdom, SE1 7EH (e-mail: massimo.garriboli@gstt.nhs.uk).
} Eur J Pediatr Surg Rep 2016;4:37-39.
Abstract
Keywords
- pediatric urology
- congenital anomaly
- urethral duplication

Duplication of the urethra is a rare congenital anomaly, with approximately 300 cases reported in the literature. We report a unique case of this condition in a male infant. This case differs from the classical Effman type II-A2 duplication because of the presence of two hypospadic urethral meati, as opposed to a ventral or dorsal accessory meatus with a normally positioned distal urethra. The patient underwent a single-stage repair consisting of a proximal urethra-urethral anastomosis and distal urethral tubularization at 21 months of age with excellent results in terms of both function and cosmesis.

\section{New Insights and Importance for the Pediatric Surgeon}

This report demonstrates a new variant of urethral duplication of which pediatric surgeons should be aware. In cases of proximal hypospadias, the surgeon should consider the possibility of a distal accessory urethra, which may require consideration when planning surgery. This case highlights that this unusual variant can be successfully managed in a single-stage procedure with good functional and cosmetic results.

\section{Introduction}

Urethral duplication is a rare, congenital lower urinary tract anomaly. Although the exact embryological mechanism for this condition remains unclear, it is thought to relate to a disruption of development of the lateral folds of Rathke during hindgut development. ${ }^{1}$ Several anatomical variants have been described, and the classification system proposed by Effman, Lebowitz, and Colodny has been widely adopted ${ }^{2}$ ( - Fig. 1 ).

Some of these lesions may be totally asymptomatic; obviating the need for surgery. However, the more complex variants may require multiple operations to obtain functionality and these interventions entrain future risks of incontinence and stricture. $^{3-5}$ In this case we describe the management of a urethral duplication with two hypospadic meati in a 21month-old boy.

received

May 15, 2016

accepted after revision

July 18, 2016

published online

November 8, 2016

\section{Case Report}

A 27-week premature boy was referred to the pediatric urology clinic at the age of 9 months for assessment of a proximal hypospadias. He had a complicated neonatal course; spending 11 weeks in the neonatal intensive care unit, initially requiring a ventilator. He had neonatal complications of jaundice requiring phototherapy, and necrotizing enterocolitis, which was managed medically.

Examination revealed a well-developing boy with a proximal penile hypospadias. However, closer inspection revealed what appeared to be a second urethral meatus opening within the glans (-Fig. 2A).

A preoperative contrast study ( - Fig. 3) revealed two distinct urethral channels originating as a single channel from the bladder neck and then running separately-by

\footnotetext{
(c) 2016 Georg Thieme Verlag KG Stuttgart · New York
}

License terms

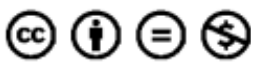

10.1055/s-0036-1588015. ISSN 2194-7619. 

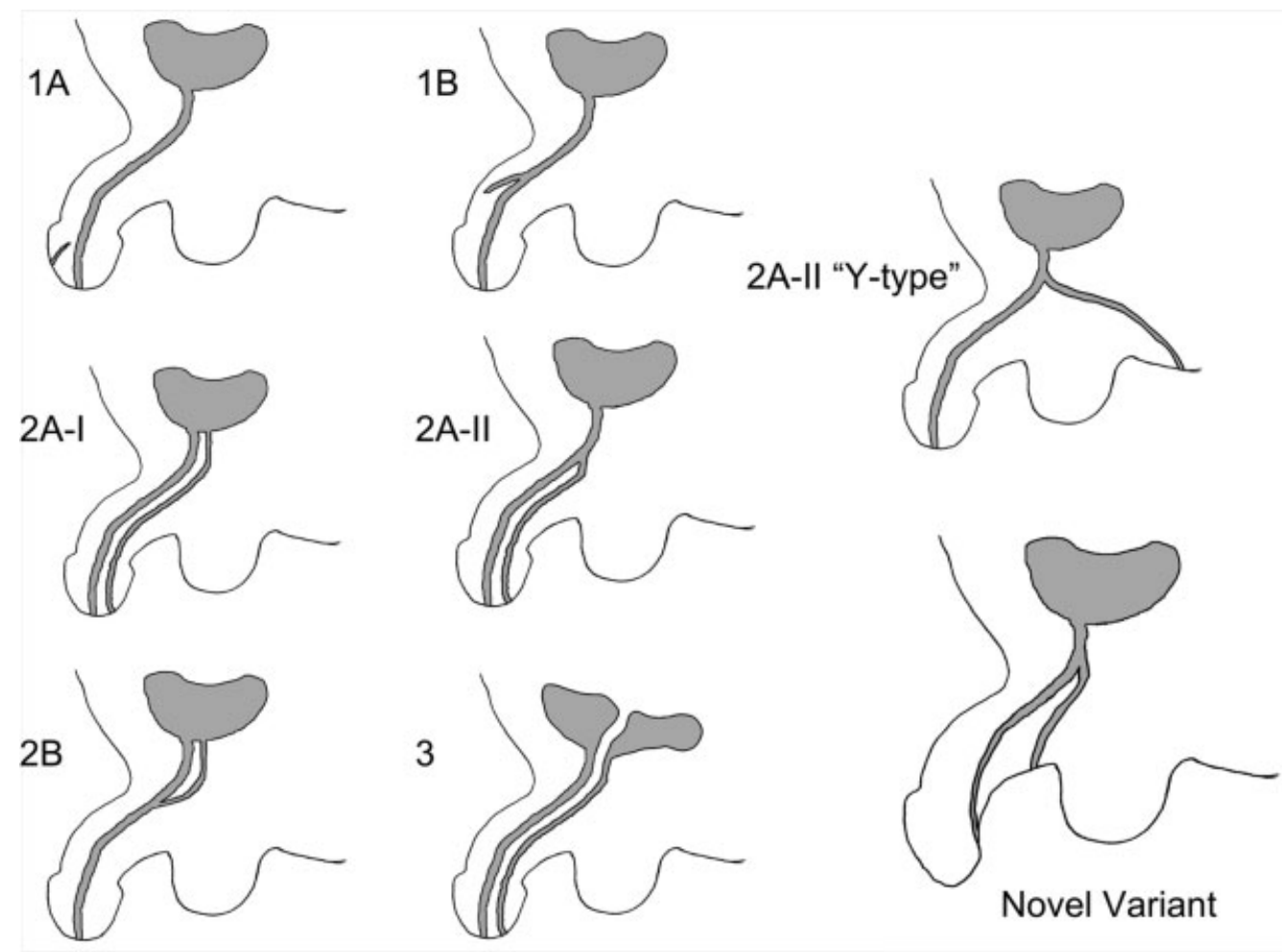

Fig. 1 Effman classification of urethral duplication with described 'novel' variant (adapted from Effman et al ${ }^{2}$ ). • Type 1: 'Blind ending channels' or incomplete duplication $\circ$ Type 1A: A blind ending channel opening on the dorsal or ventral surface of the penis in the midline, without communicating with either the bladder or urethra. $\circ$ Type 1B: A blind ending channel originating from the urethra. $\bullet$ Type 2: Patent and complete duplication $\circ$ Type 2A: Two urethral meati (which may open anywhere along the midline). $\mathbf{2 A - I}$ : The two urethrae originate separately from the bladder. 2A-II: The accessory urethra divides from the main urethra and maintains a separate course $2 \mathrm{~A}$-II Y-type: the ventral urethra opens in the perineum $\circ$ Type 2B: The two urethrae unite and form a single channel before opening at the skin $\bullet$ Type 3: Urethral duplication associated with caudal duplication (i.e., duplication of the bladder)

definition an Effman Type 2A-II. Surgery was performed at the age of 21 months: the challenge was the presence of a hypospadic rudimentary dorsal urethra, chordee, and a hooded foreskin ( - Fig. 2A). A complete penile degloving was performed to correct the chordee ( - Fig. 2B), the dorsal urethra was opened in the midline from the meatus to the level of the proximal urethral ending.

The bridge between proximal urethra and duplicated urethra was divided and a urethra-urethroplasty was performed A complete distal urethral tubularization, layered closure, and glanuloplasty resulted in a single, glanular urethral opening ( - Fig. 2C). A urinary catheter was left in place for 1 week and then removed without complication.

At follow-up 21 months postoperatively, there was a good cosmetic result with no postsurgical complications ( - Fig. 2D). The mother reported that her son was passing urine with a thick and straight stream with no evidence of fistula.

\section{Discussion}

Various theories to explain the origin of a urethral duplication have been suggested, with different types of classification proposed, ${ }^{6-8}$ the most widely adopted being that proposed by Effman. ${ }^{2}$ Of the cases of urethral duplication reported in the literature, type I lesions are thought to be the most common, although since these are generally asymptomatic, their true incidence is unknown. ${ }^{2}$ There have been cases reported that are associated with a single hypospadic urethra, commonly associated to an atretic accessory channel distally, such as in type $1 \mathrm{~A}$ lesions. ${ }^{9,10}$

The type 2 A-II group is characterized by the presence of a second urethra which divides from the main urethra, and maintains a separate course. This group includes a subgroup of patients in whom the ventral urethra opens in the perineum ("Y-type" duplication) (-Fig. 1).

In our patient the ventral urethra opened at the base of the shaft, thus representing a variant that could be considered an intermediate between the classic type 2 A-II and the "Y-type" duplication. As in the majority of patients with "Y-type" duplication, the ventral urethra was the more functional, while the dorsal urethra was less developed.

Several established techniques have been described for the correction of a duplicated urethra. ${ }^{5,11,12} \mathrm{~A}$ general consensus is that each patient should be considered individually, with no standard fit-all approach really being suitable for all cases of a particular lesion. ${ }^{13}$ Some authors would tend toward using the orthotopic urethra, even if it is hypoplastic; Ortolano and Nasrallah first proposed progressive urethral dilation to achieve adequate caliber. ${ }^{14}$ We believe, as suggested by Salle et al, that after dilatation of the accessory urethra the risk of inadequate 


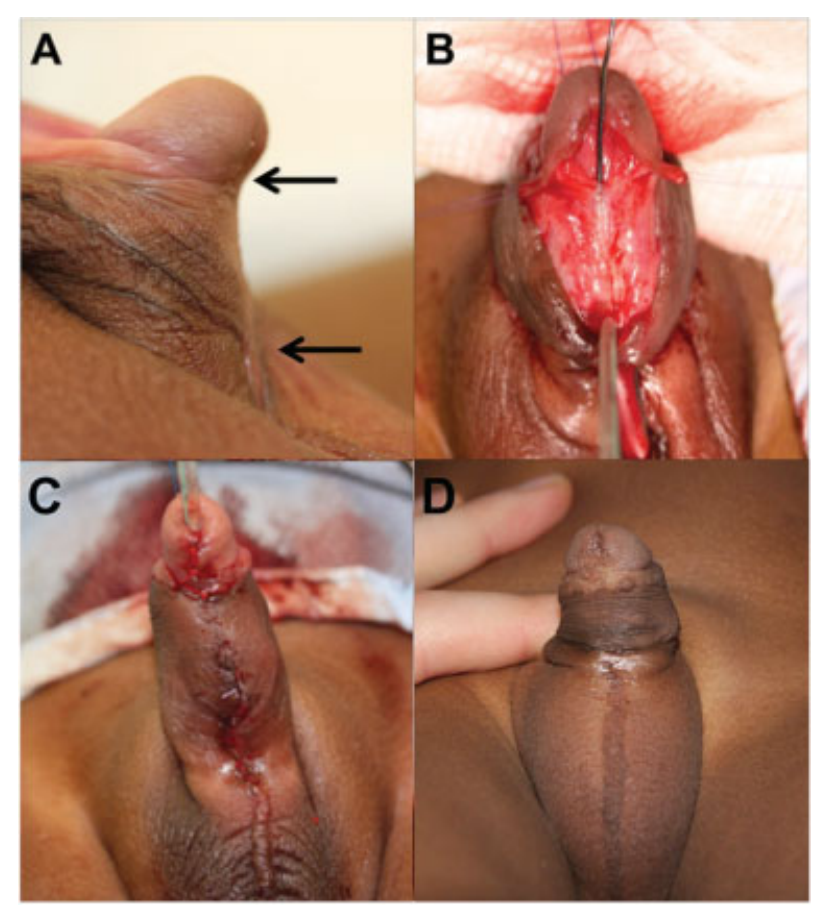

Fig. 2 Operative procedure. (A) Preoperative picture (the arrows show the position of the two urethral meati). (B) Intraoperative picture after penile degloving, the dorsal urethra is opened in the midline. (C) Immediate postoperative result. (D) Follow-up 21 months postoperatively.

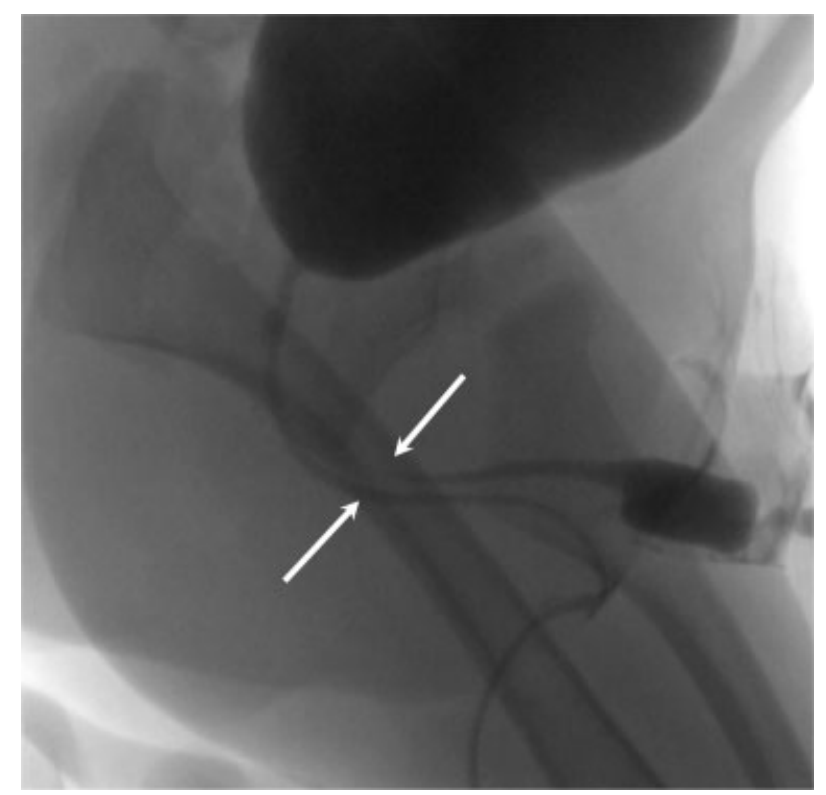

Fig. 3 A micturating cystourethrogram demonstrating a single distinct channel arising from the bladder, splitting into two (arrowed).

urine flow is high. ${ }^{10}$ Therefore in the case described, we opened the dorsal urethra and performed a tubularization over a $8 \mathrm{Fr}$ catheter, as in the classic repair for hypospadias.
The use of a voiding cystourethrogram is typically adequate to trace the course and caliber of the two channels, however, retrograde contrast may be required in cases (as the one presented) with a hypoplastic urethral opening. ${ }^{15}$

Postsurgical complications tend to manifest as stricture at the anastomosis or related to the hypoplastic channel. These may present as bladder outflow obstruction and can require further surgical intervention. There have been reported cases of fistula formation much like that documented in penoscrotal hypospadias repair. ${ }^{5,15}$

We believe this case of duplicated urethra is unique for the particular anatomy of the two urethral channels, with the main urethra being the one opening at the base of the shaft. A distal blind pit is common in hypospadias and should always prompt thorough examination for accessory urethra, in particular, in proximal hypospadias cases. This case also demonstrates the feasibility of single-stage repair in such patients.

\section{Conflict of Interest}

None.

\section{References}

1 Gyftopoulos K, Wolffenbuttel KP, Nijman RJ. Clinical and embryologic aspects of penile duplication and associated anomalies. Urology 2002;60(4):675-679

2 Effmann EL, Lebowitz RL, Colodny AH. Duplication of the urethra. Radiology 1976;119(1):179-185

3 Mane SB, Obaidah A, Dhende NP, et al. Urethral duplication in children: our experience of eight cases. J Pediatr Urol 2009;5(5): 363-367

4 Coleman RA, Winkle DC, Borzi PA. Urethral duplication: cases of ventral and dorsal complete duplication and review of the literature. J Pediatr Urol 2010;6(2):188-191

5 Mazaheri T, Rad MV, Fareghi M, Kajbafzadeh A-M. Multiple hypoplastic duplicated urethral hydrodistension and simple end-to-end anastomosis to penoscrotal hypospadias: a novel technique. Int Urol Nephrol 2014;46(9):1729-1731

6 Gross R, Moore T. Duplication of the urethra. Report of two cases and summary of the literature. Arch Surg 1950;60(4):749-761

7 Das S, Brosman SA. Duplication of the male urethra. J Urol 1977; 117(4):452-454

8 Woodhouse CR, Williams DI. Duplications of the lower urinary tract in children. Br J Urol 1979;51(6):481-487

9 Deniz N, Kirac M, Camtosun A, Irkilata L, Tan MO. Repair of hypospadiac urethral duplication with dismembered urethroplasty. Urol Int 2008;80(1):105-107

10 Salle JL, Sibai H, Rosenstein D, Brzezinski AE, Corcos J. Urethral duplication in the male: review of 16 cases. J Urol 2000;163(6): 1936-1940

11 Middleton AW Jr, Melzer RB. Duplicated urethra: an anomaly best repaired. Urology 1992;39(6):538-542

12 Podesta ML, Medel R, Castera R, Ruarte AC. Urethral duplication in children: surgical treatment and results. J Urol 1998;160(5): 1830-1833

13 AbouZeid AA, Safoury HS, Mohammad SA, et al. The double urethra: revisiting the surgical classification. Ther Adv Urol 2015;7(2):76-84

14 Ortolano V, Nasrallah PF. Urethral duplication. J Urol 1986;136(4): 909-912

15 Haleblian G, Kraklau D, Wilcox D, Duffy P, Ransley P, Mushtaq I. Ytype urethral duplication in the male. BJU Int 2006;97(3):597-602 